\title{
A NOTE ON ULTRAPRODUCTS OF VELTMAN MODELS
}

\author{
Mladen Vuković \\ University of Zagreb, Croatia
}

\begin{abstract}
We consider ultraproducts of Veltman models, and show that a version of Łos theorem is true.
\end{abstract}

\section{INTRODUCTION}

Interpretability logic is an extension of provability logic GL (Gödel, Löb). For precise definitions and details, see e.g. [5] or [6]. We are only interested in interpretability logic as a system of modal logic, i.e., we are interested in semantics for interpretability logic. There are several kinds of semantics for interpretability logic. The basic semantics is given by Veltman models. In [1] ultraproducts are used for a proof of the existence of countably saturated models and for modal definability. In [4] various preservation results, i.e., versions of Łos theorem, for ultraproducts of Kripke models are considered.

We define an ultraproduct of Veltman models over a countably complete ultrafilter. Then we show that the ultraproduct is a Veltman model. Using the standard translation for interpretability logic we prove a version of Łos theorem for Veltman models.

\section{Ultraproducts of Veltman MOdels}

The notion of Veltman model is defined in [3].

Definition 2.1. An ordered triple $\left\langle W, R,\left\{S_{w}: w \in W\right\}\right\rangle$ is called a Veltman frame if it satisfies the following conditions:

a) $\langle W, R\rangle$ is a GL-frame, i.e., $W$ is a non-empty set, and $R$ is transitive and reverse well-founded relation on $W$;

b) For every $w \in W$ is $S_{w} \subseteq W[w] \times W[w]$, where $W[w]=\{u: w R u\}$;

2010 Mathematics Subject Classification. 03F45, 03B45.

Key words and phrases. Interpretability logic, Veltman models, ultraproducts. 
c) The relation $S_{w}$ is reflexive and transitive for every $w \in W$;

d) If $w R u R v$ then $u S_{w} v$.

An ordered quadruple $\left\langle W, R,\left\{S_{w}: w \in W\right\}, \Vdash\right\rangle$ is called a Veltman model if it satisfies the following conditions:

1) $\left\langle W, R,\left\{S_{w}: w \in W\right\}\right\rangle$ is a Veltman frame;

2) $\Vdash$ is a forcing relation. We emphasize only the definition

$w \Vdash A \triangleright B$ if and only if $\forall u\left((w R u \& u \Vdash A) \Rightarrow \exists v\left(u S_{w} v \& v \Vdash B\right)\right)$.

We denote a Veltman model $\left\langle W, R,\left\{S_{w}: w \in W\right\}, \Vdash\right\rangle$ shortly by $W$.

Definition 2.2 (see [2]). An ultrafilter $U$ is countably complete if for each sequence $\left(A_{n}\right)_{n \in \mathbb{N}}$ in $U$ we have $\cap A_{n} \in U$.

LEMma 2.3. An ultrafilter $U$ is countably complete if and only if there is no sequence $\left(B_{n}\right)_{n \in \mathbb{N}}$ in $U$ such that $\cap B_{n}=\emptyset$.

Proof of Lemma 2.3. If $U$ is an ultrafilter such that there is a sequence $\left(B_{n}\right)$ such that $\cap B_{n}=\emptyset$, then $\cap B_{n} \notin U$.

Let $\left(A_{n}\right)$ be a sequence in an ultrafilter $U$ such that $\cap A_{n} \notin U$. Then we have $\left(\cap A_{n}\right)^{c} \in U$. Let us define a sequence $\left(B_{n}\right)$ by: $B_{0}=\left(\cap A_{n}\right)^{c}$, and $B_{n+1}=A_{n}$. Obviously, $B_{n} \in U$ for each $n \in \mathbb{N}$, and $\cap B_{n}=\emptyset$.

Let $\left\{W_{i}: i \in I\right\}$ be a set of Veltman models, $W_{i}=\left(W_{i}, R_{i},\left\{S_{w}^{(i)}\right.\right.$ : $\left.\left.w \in W_{i}\right\}, \Vdash\right)$. Let $U$ be a countably complete ultrafilter over the set $I$, and $W=\prod_{U} W_{i}$. We define a relation $R \subseteq W \times W$ in the following way:

$\bar{f} R \bar{g}$ if and only if $\left\{i \in I: f(i) R_{i} g(i)\right\} \in U$.

Let us denote $W[\bar{g}]=\{\bar{f}: \bar{g} R \bar{f}\}$, for each $g \in \prod_{i \in I} W_{i}$. For every $\bar{g} \in W$ we define a relation $S_{\bar{g}} \subseteq W[\bar{g}] \times W[\bar{g}]$ by

$$
\bar{f} S_{\bar{g}} \bar{h} \text { if and only if }\left\{i \in I: f(i) S_{g(i)}^{(i)} h(i)\right\} \in U .
$$

Finally, we define a forcing relation on the set $W$ by:

$$
W, \bar{f} \Vdash p \text { if and only if }\left\{i \in I: W_{i}, f(i) \Vdash p\right\} \in U \text {. }
$$

Proposition 2.4. The ultraproduct of Veltman models over a countably complete ultrafilter is a Veltman model.

Proof of Proposition 2.4. It is easy to check that the relation $R$ is transitive. Let us suppose that the relation $R$ is not reverse well-founded. Let $\left(\bar{f}_{n}\right)$ be a sequence in $W$ such that $\bar{f}_{n} R \bar{f}_{n+1}$. Then for each $n \in \mathbb{N}$ we have $A_{n}:=\left\{i \in I: f_{n}(i) R_{i} f_{n+1}(i)\right\} \in U$. Because $U$ is a countably complete ultrafilter, then $\cap A_{n} \neq \emptyset$. Let $i$ be an arbitrary element of the set $\cap A_{n}$. Then we have $f_{1}(i) R_{i} f_{2}(i) R_{i} f_{3}(i) \ldots$ So, we have that the relation $R_{i}$ is not reverse well-founded, which is a contradiction. 
It is easy to check that the relation $S_{\bar{g}}$ is reflexive and transitive, for each $g \in \prod_{i \in I} W_{i}$. Let us show that $\bar{g} R \bar{f} R \bar{h}$ implies $\bar{f} S_{\bar{g}} \bar{h}$. Let $f, g$, and $h$ be elements of $\prod_{i \in I} W_{i}$ such that $\bar{g} R \bar{f} R \bar{h}$. Then we have

$$
\left\{i \in I: g(i) R_{i} f(i)\right\} \in U \quad \text { and } \quad\left\{i \in I: f(i) R_{i} h(i)\right\} \in U,
$$

This implies $\left\{i \in I: g(i) R_{i} f(i)\right\} \cap\left\{i \in I: f(i) R_{i} h(i)\right\} \in U$, i.e., $\{i \in I$ : $\left.g(i) R_{i} f(i) R_{i} h(i)\right\} \in U$. Because $W_{i}$ is a Veltman model, then we have

$$
\left\{i \in I: g(i) R_{i} f(i) R_{i} h(i)\right\} \subseteq\left\{i \in I: f(i) S_{g(i)}^{(i)} h(i)\right\} .
$$

So, $\left\{i \in I: f(i) S_{g(i)}^{(i)} h(i)\right\} \in U$, i.e., $\bar{f} S_{\bar{g}} \bar{h}$.

\section{Standard TRANSlations}

Standard translation is a function that maps each modal formula to a first-order formula. In [1] standard translation of "standard" modal logic is considered. Interpretability logics are nonstandard logics. We define a standard translation for interpretability logics, and show basics results. Let $\sigma=\left\{P_{0}, P_{1}, \ldots\right\} \cup\{R, S\}$ be a first-order signature, where $P_{i}$ is an unary relation symbol, $R$ is a binary relation symbol, and $S$ is a ternary relation symbol.

DeFinition 3.1. Let $x$ be a first-order variable. The standard translation $S T_{x}$ taking modal formulas to first-order $\sigma$-formulas is defined as follows:

$$
\begin{aligned}
& S T_{x}\left(p_{i}\right)=P_{i}(x), \\
& S T_{x}(\neg \varphi)=\neg S T_{x}(\varphi), \\
& S T_{x}(\varphi \wedge \psi)=S T_{x}(\varphi) \wedge S T_{x}(\psi), \\
& S T_{x}(\square \varphi)=\forall y\left(x R y \rightarrow S T_{y}(\varphi)\right), \\
& S T_{x}(\varphi \triangleright \psi)=\forall y\left(x R y \wedge S T_{y}(\varphi), \rightarrow \exists z\left(S(x, y, z) \wedge S T_{z}(\psi)\right)\right) .
\end{aligned}
$$

The following proposition is easy to prove by induction on complexity of modal formula.

Proposition 3.2. Let $\varphi$ be a modal formula, $W$ a Veltman model and $w \in W$. Then we have:

$$
W, w \Vdash \varphi \quad \text { if and only if } \quad W \models S T_{x}(\varphi)[w] .
$$

By using the previous proposition and Łos theorem (see [2]), we get the following result.

Corollary 3.3. Let $\left\{W_{i}: i \in I\right\}$ be a set of Veltman models, $U$ a countably complete ultrafilter over a set $I$, and $\varphi$ a modal formula. For each $f \in \prod_{i \in I} W_{i}$ we have:

$$
\prod_{U} W_{i}, \bar{f} \Vdash \varphi \quad \text { if and only if } \quad\left\{i \in I: W_{i}, f(i) \Vdash \varphi\right\} \in U .
$$


Corollary 3.4. Let $\left(W, R,\left\{S_{w}: w \in W\right\}, \Vdash\right)$ be a Veltman model, $U$ a countably complete ultrafilter over a set $I$, and $w \in W$. Let $f_{w}: I \rightarrow W$ be a function that is defined by $f_{w}(i)=w$. For each modal formula $\varphi$ we have:

$$
W, w \Vdash \varphi \quad \text { if and only if } \quad \prod_{U} W, \overline{f_{w}} \Vdash \varphi \text {. }
$$

REMARK 3.5. We would like to mention that we have not obtained analogous results for generalized Veltman semantics. The definition of generalized Veltman model is given for example in [7]. It is easy to define a notion of ultraproduct for generalized Veltman model, and prove quasireflexivity of the relation $S_{\bar{g}}$. However we have not been able to produce a proof that the relation $S_{\bar{g}}$ is quasi-transitive.

REMARK 3.6. We have mentioned in introduction that ultraproducts are useful for a proof of existence of countably saturated models. Each countably saturated model is a modal saturated Kripke model (see [1]). We try to prove van Benthem characterization theorem for interpretabilty logics. A big problem is to define a notion of modal saturated Veltman model, and to prove that an ultrafilter extension of Veltman model is Veltman model.

ACKNOWLEDGEMENTS.

The author would like to express his gratitude to Mirna Džamonja for a valuable comment.

\section{REFERENCES}

[1] P. Blackburn, M. de Rijke and Y. Venema, Modal logic, Cambridge University Press, Cambridge, 2001.

[2] C. C. Chang, H. J. Keisler, Model theory, North-Holand, 1990.

[3] D. de Jongh, F. Veltman, Provability logics for relative interpretability, in: Mathematical Logic, Proceedings of the 1988 Heyting Conference, ed. P. P. Petkov, Plenum Press, New York, 1990, 31-42.

[4] V. Goranko, M. Otto, Model theory of modal logic, in: Handbook of Modal Logic, eds. F. Wolter et al., Elsevier, 2006.

[5] G. Japaridze, D. de Jongh, The logic of provability, in: Handbook of Proof Theory, ed. S. R. Buss, Elsevier, 1998, 475-546.

[6] A. Visser, An overview of interpretability logic, in: Advances in modal logic, Vol. 1 (Berlin, 1996), 307359, CSLI Lecture Notes 87, CSLI Publ., Stanford, USA, 1998.

[7] D. Vrgoč, M. Vuković, Bisimulations and bisimulation quotients of generalized Veltman models, Logic Jou. IGPL 18 (2010), 870-880.

M. Vuković

Department of Mathematics

University of Zagreb

10000 Zagreb

Croatia

E-mail: vukovic@math.hr

Received: 20.12.2009. 